\title{
The Yamabe invariant for axially symmetric initial data of two Kerr black holes
}

\author{
Gastón A Avila ${ }^{1}$ and Sergio Dain ${ }^{1,2}$ \\ ${ }^{1}$ Facultad de Matemática, Astronomía y Física, Universidad Nacional de Córdoba, \\ Ciudad Universitaria, 5000 Córdoba, Argentina \\ ${ }^{2}$ Max Planck Institute for Gravitational Physics (Albert Einstein Institute), Am Mühlenberg 1, \\ D-14476 Potsdam, Germany
}

Received 10 June 2008, in final form 6 August 2008 Published 23 October 2008

Online at stacks.iop.org/CQG/25/225002

\begin{abstract}
An explicit three-dimensional Riemannian metric is constructed which can be interpreted as the (conformal) sum of two Kerr black holes with aligned angular momenta. When the separation distance between them is large we prove that this metric has a positive Ricci scalar and hence a positive Yamabe invariant. This metric can be used to construct axially symmetric initial data for two Kerr black holes with large total angular momentum.
\end{abstract}

PACS numbers: 04.20.Ex, 04.25.dg, 04.70.Bw

\section{Introduction}

The numerical study of the binary black hole problem has recently made significant progress $[1,6,21]$. It is now possible to calculate the evolution of a wide variety of astrophysical scenarios. In order to do this, appropriate initial data for the Einstein equations are necessary, such that they describe the initial conditions of these systems.

A natural physical requirement for binary black hole initial data is that in the limit of large separation each of the black holes approximates a stationary, isolated, black hole (in vacuum, this implies that the black hole approximates the Kerr black hole). Otherwise, the data will contain spurious radiation which can in principle contaminate the final waveform (see [17]).

The nonlinearity of the constraint equations makes the problem of constructing initial data satisfying such a far limit a nontrivial one. In [11] such data for two Kerr black holes with a far limit has been constructed. The procedure uses the conformal method for solving the constraint equations (see the review article [2] and references therein). In this construction, one starts with a superposition of two conformal Kerr metrics to get a new conformal metric, which is then used as a 'seed' metric for the conformal method. Then, a conformal factor is calculated such that the corresponding rescaled metric satisfies the constraint equations. The existence and uniqueness of that conformal factor can be proved provided the 'seed' metric satisfies an important requirement: its Yamabe invariant must be positive (see [2] for 
a discussion about the Yamabe invariant in the context of the constraint equations). In order to ensure this condition in [11], it has been assumed that the individual angular momentum parameters of the black holes are small with respect to the masses.

This assumption is, of course, an undesirable restriction. Highly spinning black holes are relevant in many astrophysical situations. Remarkable examples of these are the large merger recoil kicks (see $[5,7,15,16,18,19])$.

The purpose of this paper is to overcome this restriction for the axially symmetric case. We provide an explicit metric, having three 'ends', which is constructed as a superposition of two Kerr black hole metrics with aligned or anti-aligned angular momenta. When the mass parameters of one black hole is zero, the metric reduces exactly to a Kerr metric. The important property of this metric is that when the separation distance between the black holes is large enough its Ricci scalar is positive. The parameters of the individual Kerr black holes are only restricted by the Kerr inequality $|a| \leqslant m$, where $a$ is the angular momentum per mass unit and $m$ is the mass of the individual black hole. That is, the angular momentum is not assumed to be small. Instead, we assumed the physical reasonable condition that the separation distance between them is large. In this sense, we say that the metric satisfies the far limit. This constitutes the main result of the paper.

When the three 'ends' are asymptotically flat (the non-extreme cases) the positivity of the Ricci scalar implies the positivity of the corresponding Yamabe invariant. And hence the metric can be used as a seed metric for the construction mentioned above. In this way axially symmetric initial data can be constructed for two Kerr-like black holes with large angular momentum. However, we emphasize that our result also covers the extreme limit $|a|=m$ of any of the black holes. We believe that this can be used to construct (the still unknown) initial data for two extreme Kerr black holes.

\section{Main result}

Consider the Kerr black hole (i.e. the Kerr metric such that $|a| \leqslant m$ ) in Boyer-Lindquist coordinates. Take any constant $t$ slice in these coordinates and denote by $\tilde{h}_{i j}$ the intrinsic three-dimensional metric of the slice. There exist spatial coordinates $(\rho, z, \phi)$ on the slice such that the metric $\tilde{h}_{i j}$ has the following form:

$$
\tilde{h}_{i j}=(1+u)^{4} h_{i j}
$$

where

$$
h_{i j}=e^{2 q}\left(\mathrm{~d} \rho^{2}+\mathrm{d} z^{2}\right)+\rho^{2} \mathrm{~d} \phi^{2},
$$

and the functions $q$ and $u$ (which do not depend on the coordinate $\phi$ ) are explicitly given by equations (A.1) in the appendix. The slice is a three-dimensional manifold $M$ which has the topology $M=\mathbb{R}^{3} \backslash\{0\}$, where $\{0\}$ denotes the origin in the coordinates $(\rho, z, \phi)$. For the results presented here a relevant property of the Kerr metric is that $u \geqslant 0$ (see lemma 3.1).

We construct a new manifold $S$ by removing two points from $\mathbb{R}^{3}$ denoted by $s_{1}$ and $s_{2}$, namely $S=\mathbb{R}^{3} \backslash\left\{s_{1}, s_{2}\right\}$. Let $(\rho, z, \phi)$ be cylindrical coordinates on $S$ such that the two points $s_{1}$ and $s_{2}$ are located at the axis $\rho=0$, separated by coordinate distance $d$. This requirement does not completely fix the coordinate system, since there still exists a translation freedom in the $z$-coordinate $z \rightarrow z+c$, where $c$ is any arbitrary constant. Later on we will make use of this freedom to simplify the computations. However, for the formulation of the results there is no loss of generality if we chose the coordinate system such that $s_{1}$ is located at $d / 2$ and $s_{2}$ at $-d / 2$ on the $z$-coordinate axis. 
Given the functions $q\left(\rho, z ; m_{1}, a_{1}\right)$ and $u\left(\rho, z ; m_{1}, a_{1}\right)$ of the Kerr initial data, with parameters $\left(a_{1}, m_{1}\right)$, we define the new functions $\left(q_{1}, u_{1}\right)$ by

$$
q_{1}=q\left(\rho, z-d / 2 ; m_{1}, a_{1}\right), \quad u_{1}=u\left(\rho, z-d / 2 ; m_{1}, a_{1}\right) .
$$

In the same way, for functions $q\left(\rho, z, m_{2}, a_{2}\right)$ and $u\left(\rho, z, m_{2}, a_{2}\right)$ we define $\left(q_{2}, u_{2}\right)$ by

$$
q_{2}=q\left(\rho, z+d / 2 ; m_{2}, a_{2}\right), \quad u_{2}=u\left(\rho, z+d / 2 ; m_{2}, a_{2}\right) .
$$

With this set of functions constructed from the Kerr metric, we define a metric on $S$, depending on the parameters $\left(d, m_{1}, a_{1}, m_{2}, a_{2}\right)$, by

$$
\tilde{H}_{i j}=\left(1+u_{1}+u_{2}\right)^{4} H_{i j}
$$

where

$$
H_{i j}=e^{2\left(q_{1}+q_{2}\right)}\left(\mathrm{d} \rho^{2}+\mathrm{d} z^{2}\right)+\rho^{2} \mathrm{~d} \phi^{2} .
$$

Since $u_{i}$ and $u_{2}$ are positive functions, and furthermore $q_{1}$ and $q_{2}$ are bounded, $\tilde{H}_{i j}$ is well defined as a metric on $S$ for any choice of the parameter $d$.

For the non-extreme case (i.e. $\left|a_{1}\right|<m_{2},\left|a_{2}\right|<m_{2}$ ) one can check that the metric $\tilde{H}_{i j}$ is asymptotically flat with three asymptotic ends. These ends are denoted by $s_{\infty}$, which corresponds to the limit $r \rightarrow \infty$, where $r=\sqrt{\rho^{2}+z^{2}}$ and $s_{1}, s_{2}$, which correspond to the limits $r_{1} \rightarrow 0$ and $r_{2} \rightarrow 0$ respectively, where $r_{1}=\sqrt{\rho^{2}+(z-d / 2)^{2}}$ and $r_{2}=\sqrt{\rho^{2}+(z+d / 2)^{2}}$. The total mass of the metric (5) at the end $s_{\infty}$ is given by $m_{1}+m_{2}$.

If one of the ends is extreme (that is $\left|a_{1}\right|=m_{1}$ or $\left|a_{2}\right|=m_{2}$ ), then the metric will still be asymptotically flat at the end $s_{\infty}$ but will not be asymptotically flat at the extreme end.

Denote by $R_{\tilde{H}}$ the Ricci scalar of the metric (5). The following theorem constitutes the main result of this paper.

Theorem 2.1. There exists a constant $d_{c}$ such that if $d \geqslant d_{c}$ then $R_{\tilde{H}} \geqslant 0$ on S. Moreover, if $a_{1}$ or $a_{2}$ is different from zero, then $R_{\tilde{H}}>0$ on $S$.

For $a_{1}=a_{2}=0$ we have $R_{\tilde{H}}=0$. In this limit the metric reduces to the BrillLindquist metric [4]. If we set to zero the mass of one of the black holes (this implies that the corresponding angular momentum is also zero) then the metric (5) reduces to the Kerr metric (1).

Consider the Yamabe invariant defined on the manifold $S$ for the metric $\tilde{H}_{i j}$

$$
\lambda=\inf _{\varphi \in C_{c}^{\infty}(S), \varphi \neq 0} \frac{\int_{S}\left(8|D \varphi|^{2}+R_{\tilde{H}} \varphi^{2}\right) \mathrm{d} \mu}{\left(\int_{S}|\varphi|^{6} \mathrm{~d} \mu\right)^{1 / 3}},
$$

where $C_{c}^{\infty}(S)$ denotes the set of smooth functions with compact support in $S,|D \varphi|^{2}=$ $\tilde{H}^{i j} \partial_{i} \varphi \partial_{j} \varphi, \mathrm{d} \mu$ is the volume element with respect to $\tilde{H}_{i j}$, and $\varphi \not \equiv 0$ means that $\varphi$ cannot be identically zero everywhere.

For the non-extreme cases, theorem 2.1 implies that $\lambda>0$ (it is obvious that $\lambda \geqslant 0$; for proving that it is strictly positive we use lemma 4.1 in [8]).

The main application of theorem 2.1 is the construction of two (non-extreme) Kerr black holes data. The results proved so far assumed that the angular momentum parameter $a$ is small with respect to the mass (see [11]). Theorem 2.1 allows us to generalize these results for any $a$ such that $|a|<m$, but only for the axially symmetric case (that is, when the angular momentum of the black holes are aligned or anti-aligned). Using the conformal method and the metric (5) as a 'seed' conformal metric the complete data can be obtained as follows. Since this procedure involves standard applications of known results we only sketch the proof. First we superpose two extrinsic curvatures from two Kerr initial data. This can be done in 
general (see [11]), however in axial symmetry the procedure is much simpler and can be done explicitly (see, for example, [10, 14]). Then, we use existence theorems for the Lichnerowicz equation (see for example, $[9,11,20]$ ) to prove that there exists a new conformal factor such that a conformal rescaling of the metric $\tilde{H}_{i j}$ satisfies the vacuum constraint equations with the above constructed second fundamental form (see [11] for details). It is in this last step when theorem 2.1 plays an important role. In order to apply these existence theorems we need to ensure that the conformal metric has positive Yamabe invariant. Using theorem 2.1 we only require that the separation distance between the black holes is large. We also note that although this procedure is identical to that proposed in [11], the 'seed' conformal metric is different.

There is no unique way of constructing the 'sum' of two Kerr metrics. In particular, the metric used in [11] is not in the same conformal class as that used here (that is, they are not related by a conformal factor). Theorem 2.1 suggests that the conformal metric (6) is more natural than that used in [10], at least for the axially symmetric case.

The above construction applies to the non-extreme case. Remarkably, theorem 2.1 is also valid for the extreme case $|a|=m$. It is very likely that this theorem can also be used to construct a superposition of two extreme Kerr black holes. But this remains to be seen. The existence results for the Lichnerowicz equations proved so far in the literature applies only to asymptotically flat manifolds and not to manifolds with cylindrical ends like extreme Kerr.

\section{Properties of Kerr initial data}

In this section we establish the three key properties of Kerr intrinsic metric which allow us to prove theorem 2.1 in the following section. These properties are collected in lemmas 3.1-3.3.

We consider the Kerr metric $\tilde{h}_{i j}$ given by (1), on the manifold $M=\mathbb{R}^{3} \backslash\{0\}$, where the functions $u$ and $q$ are given by (A.1). The Ricci scalar of $\tilde{h}_{i j}$ is denoted by $\tilde{R}$ and the Ricci scalar of the conformal metric $h_{i j}$ (defined by (2)) is denoted by $R$. We emphasize that all the functions involved are smooth on $M$.

Lemma 3.1. Assume $|a| \leqslant m$ and $m \neq 0$. Then, we have that $u>0$ everywhere on $M$.

Proof. We prove this by explicitly showing that $\psi>1$ if $m \neq 0$, where $\psi=1+u$. Using (A.1) and (A.4) we obtain

$$
\psi^{4} \geqslant \frac{\tilde{r}^{2}}{r^{2}}
$$

We use equation (A.5) for $\tilde{r}$ to obtain

$$
\frac{\tilde{r}}{r}=1+\frac{m}{r}+\frac{m^{2}-a^{2}}{4 r^{2}} \geqslant 1+\frac{m}{r} .
$$

Using (8) and (9) we get our final inequality

$$
\psi \geqslant \sqrt{1+\frac{m}{r}}
$$

We also mention the following upper bound for $\psi$ that can be obtained from equation (A.3)

$$
\psi^{2} \leqslant \frac{\tilde{r}}{r}\left(1+\frac{a^{2}}{\tilde{r}^{2}}\right),
$$


for the extreme case $|a|=m$ this reduces to

$$
\psi^{2} \leqslant\left(1+\frac{m}{r}\right)\left(1+\frac{m^{2}}{(r+m)^{2}}\right) .
$$

These bounds are sharp at infinity, in the sense that the right-hand side of (11) and (12) goes to 1 as $r \rightarrow \infty$.

Lemma 3.2. Assume $|a| \leqslant m$ and $a \neq 0$. Then, $\tilde{R} \geqslant 0$ on $M$, and $\tilde{R}=0$ only at $\rho=0$.

Proof. The first statement is immediate if one considers the Hamiltonian constraint equation in the given slice and the fact that these slices are maximal (i.e. the trace of the second fundamental form is zero). In effect, since $\tilde{K}=\tilde{K}_{i j} \tilde{h}^{i j}=0$ (where $\tilde{K}_{i j}$ is the second fundamental form of the slice) the Hamiltonian constraint is given by

$$
\tilde{R}=\tilde{K}_{i j} \tilde{K}^{i j} \geqslant 0 \text {. }
$$

To prove the second part of the theorem we compute the right-hand side of (13). The Kerr second fundamental form $\tilde{K}_{i j}$ can be expressed in terms of derivatives of a potential $\omega$ (see, for example, $[12,13])$. In particular, for the square of $\tilde{K}_{i j}$ we have

$$
\tilde{K}_{i j} \tilde{K}^{i j}=\frac{\mathrm{e}^{-2 q}|\partial \omega|^{2}}{2 \psi^{4} X^{2}} .
$$

The explicit expression for $\omega$ is given in equation (A.6). We calculate

$$
|\partial \omega|^{2}=\frac{4 m^{2} a^{2} \rho^{6}}{r^{8} \Sigma^{4}} F
$$

where

$$
F=\left(4 \Delta a^{4} \tilde{r}^{2}(\sin (2 \theta))^{2}+\left(3 \tilde{r}^{4}+a^{2} \tilde{r}^{2}+a^{2}\left(\tilde{r}^{2}-a^{2}\right) \cos ^{2} \theta\right)^{2}\right)
$$

We have

$$
F \geqslant 9 \tilde{r}^{8} \text {. }
$$

Using (14) and (15) and the explicit expressions (A.1) we obtain the following lower bound:

$$
\tilde{R} \geqslant \frac{18 m^{2} a^{2} \tilde{r}^{8} \sin ^{2} \theta}{\left(\tilde{r}^{2}+a^{2}\right)^{7}},
$$

which proves the theorem because $\tilde{r} \geqslant m$. Note that for the extreme case this bound reduces to

$$
\tilde{R} \geqslant \frac{18 m^{4}(r+m)^{8} \sin ^{2} \theta}{\left((r+m)^{2}+m^{2}\right)^{7}} .
$$

As a side remark we point out that the bounds (18) and (19) are sharp in the limit $r \rightarrow 0$ and $r \rightarrow \infty$.

For the following lemma we define the function $R^{-}$to be the negative part of $R$

$$
R^{-}=\min \{0, R\} \text {. }
$$

Lemma 3.3. Assume $|a|<m$. Then the function $R^{-}$has compact support in M. Moreover, the support of $R^{-}$does not intersect the $\rho=0$ axis (see figure 1 ).

Proof. Take a small ball centered at the origin. The leading-order term in the asymptotic expansion of $R$ for $r \rightarrow 0$ (see equation (A.11)) is positive. Hence, there exists a constant $r_{-}$ such that the function $R$ is positive in small ball of radius $r_{-}$centered at the origin. We also 


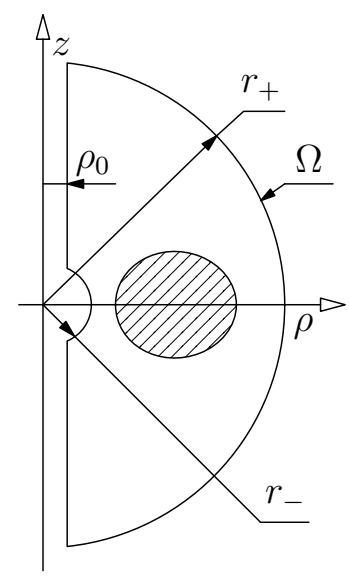

Figure 1. Non-extreme case: the region $\Omega$ contains the support of $R^{-}$(shaded region).

have that $R$ is positive evaluated at the axis $\rho=0$ (see equation (A.29)). Then, there exists a small cylinder of radius $\rho_{0}$ such that $R$ is positive outside this cylinder.

In a similar fashion, the leading-order term in the asymptotic expansion of $R$ for $r \rightarrow \infty$ (see equation (A.10)) is positive. Hence, there exists a constant $r_{+}$such that $R$ is positive outside a ball of radius $r_{+}$. Bringing these together, we conclude that the support of $R^{-}$is contained in the region $\Omega$ given by

$$
\Omega=\left\{r_{-}<r<r_{+} \cap \rho_{0}<\rho\right\} .
$$

The region $\Omega$ is showed in figure 1, where we also show how the actual support of $R^{-}$looks like when obtained by explicit computation for some values of $a$ and $m$.

The region $\Omega$ defined above will be used in the following section in the following way. One point, lets say $s_{1}$, is located at the origin of $\Omega$. The other point $s_{2}$ is chosen to be outside $\Omega$, that is $d>r_{+}$. Let $\psi_{2}$ be the conformal factor of a Kerr metric with respect to $s_{2}$. Then, the upper bounds (11) and (12) imply that $\psi_{2}$ is bounded in $\Omega$ (note that $\psi_{2}$ is not bounded at $s_{2}$ ). For points in the interior of $\Omega$ we have

$$
r_{2} \geqslant d-r \geqslant d-r_{+}
$$

and then the upper bound (11) in $\Omega$ gives the following bound which depends only on $d$ and $r_{+}$:

$$
\psi_{2}^{2} \leqslant\left(1+\frac{1}{\left(d-r_{+}\right)}+\frac{m^{2}-a^{2}}{4\left(d-r_{+}\right)^{2}}\right)\left(1+\frac{a^{2}}{\left(d-r_{+}+m\right)^{2}}\right) .
$$

For the extreme case this bound reduces to

$$
\psi_{2}^{2} \leqslant\left(1+\frac{1}{\left(d-r_{+}\right)}\right)\left(1+\frac{m^{2}}{\left(d-r_{+}+m\right)^{2}}\right) .
$$

The important point is that in the limit $d \rightarrow \infty$ we have $\psi_{2} \rightarrow 1$ in $\Omega$ (and hence $u_{2} \rightarrow 0$ in $\Omega$ ).

The statement of lemma 3.3 is false for the extreme case $|a|=m$. The reason for this is that $R$ has a different behavior at the origin in that case (see equation (A.14)), which reflects the change in this limit from an asymptotically flat end to a cylindrical one. In figure 2 we show the support of $R^{-}$in the extreme case. We see that the support of $R^{-}$'touches' the 


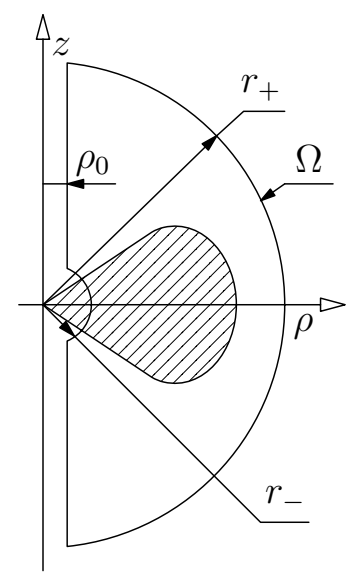

Figure 2. Extreme case: the support of $R^{-}$(shaded region) is not contained in the region $\Omega$.

origin and hence lies outside of the region $\Omega$. This is the main technical difficulty to prove theorem 2.1 in the extreme limit. To analyze that case we compute the following formula for $R$.

Lemma 3.4. Let $|a|=m$. Then, the scalar curvature $R$ is given by

$$
R=e^{-2 q}\left(-\frac{3|\partial \omega|^{2}}{2 X^{2}}+\frac{8|\partial \psi|^{2}}{\psi^{2}}\right)
$$

Proof. Define $\sigma$ by $\psi^{4}=e^{\sigma}$. The metrics $\tilde{h}_{i j}$ and $h_{i j}$ are related by the conformal transformation $\tilde{h}_{i j}=e^{\sigma} h_{i j}$, hence the relation between the corresponding scalars curvatures $\tilde{R}$ and $R$ is given by

$$
R=\tilde{R} e^{\sigma}+2 e^{-2 q} \hat{\Delta} \sigma+\frac{1}{2} e^{-2 q}|\partial \sigma|^{2},
$$

where $\hat{\Delta}$ is the flat Laplacian in three dimensions. The important step is that the Kerr metric satisfies the stationary and axially symmetric equations. For the extreme case, these equations imply (see [13])

$$
\hat{\Delta} \sigma=-\frac{|\partial \omega|^{2}}{X^{2}}
$$

We emphasize that in our coordinates equation (27) is valid only for the extreme case (see the discussion in [13]).

Combining (26), (27) and the expression for $\tilde{R}$ which comes from the Hamiltonian constraint equations (13) and (14) the result follows.

\section{Proof of the main result}

The important feature of the metric (5) is that its Ricci scalar has a simple decomposition in terms of the Ricci scalars of each individual Kerr metric, namely

$$
R_{\tilde{H}}=\frac{\psi_{1}^{5} e^{-2 q_{2}}}{\Psi^{5}}\left(\tilde{R}_{1}+\frac{R_{1}}{\psi_{1}^{5}} u_{2}\right)+\frac{\psi_{2}^{5} e^{-2 q_{1}}}{\Psi^{5}}\left(\tilde{R}_{2}+\frac{R_{2}}{\psi_{2}^{5}} u_{1}\right)
$$


where $\Psi=1+u_{1}+u_{2}, \psi_{1}=1+u_{1}$ and $\psi_{2}=1+u_{2}$. Note that the two terms in (28) are symmetric with respect to the points labels 1 and 2 .

For the non-extreme case formula (28), together with previous lemmas, gives the desired result.

Theorem 4.1 (non-extreme case). Assume that $\left|a_{1}\right|<m_{1},\left|a_{2}\right|<m_{2}$. Then there exists a constant $d_{c}$ such that if $d>d_{c}$, then $R_{\tilde{H}} \geqslant 0$. Moreover, if $a_{1}$ or $a_{2}$ is different from zero then we have $R_{H}>0$ on $S$ for $d>d_{c}$.

Proof. To analyze the sign of $R_{\tilde{H}}$ we study each of the two terms in (28) separately. Since they are symmetric in 1 and 2 it is enough to analyze only one of them. If $a_{1}=a_{2}=0$ we have $R_{\tilde{H}}=0$ (see equation (A.7)) and then the conclusion of the theorem follows. Hence, in the following we will assume that either $a_{1}$ or $a_{2}$ is different from zero. Without loss of generality we take $a_{1} \neq 0$ and analyze the first term in (28).

By lemma 3.1 the factor which multiplies the parentheses on that term is positive definite. Then, we only need to analyze the sign of

$$
K_{1}:=\tilde{R}_{1}+\frac{R_{1}}{\psi_{1}^{5}} u_{2}
$$

Take a fixed coordinate system $(\rho, z, \phi)$ centered at the point $s_{1}$. In these coordinates we have $s_{2}$ located at $z=-d$. Note that, in these coordinates, the only function in (29) which depends on $d$ is $u_{2}$.

Using lemmas 3.1 and 3.2 we know that the only function which is not positive in the definition of $K_{1}$ is $R_{1}$. Let $\Omega$ be the region defined in lemma 3.3 centered at $s_{1}$, with constants $\left(\rho_{0}, r_{-}, r_{+}\right)$. Take $d$ such that $d>r_{+}$. That is, the point $s_{2}$ is not in $\Omega$. Since $R_{1}$ is positive outside $\Omega$, it follows that $K_{1}$ is positive outside $\Omega$. Note that the constants $\left(\rho_{0}, r_{-}, r_{+}\right)$in (21) do not depend on $d$.

We now turn our attention to the interior of $\Omega$. By lemma 3.2 we know that $\tilde{R}_{1}$ is strictly positive in $\Omega$. We use the upper bound (23) for $u_{2}$ in the interior of $\Omega$. We have that $u_{2} \rightarrow 0$ in $\Omega$ as $d \rightarrow \infty$. Since $\tilde{R}_{1}$ and $R_{1}$ do not depend on $d$ it follows that there exists a constant $d_{1}$ such that $K_{1}$ is positive in the interior of $\Omega$ for all $d>d_{1}$.

For $K_{2}$ we repeat this analysis to obtain a constant $d_{2}$ such that $K_{2}>0$ is positive for all $d>d_{2}$. We chose $d_{c}$ to be the maximum between $d_{1}$ and $d_{2}$, from which the conclusion of the theorem follows.

The proof in the extreme case is more delicate because lemma 3.2 is no longer valid. However, a more detailed analysis of the asymptotic expansion at the origin of the function $K_{1}$, using lemma 3.4, allows the desired extension.

Theorem 4.2 (extreme case). Assume that either $\left|a_{1}\right|=m_{1},\left|a_{2}\right|<m_{2}$ or $\left|a_{2}\right|=m_{2},\left|a_{1}\right|<$ $m_{1}$ or $\left|a_{1}\right|=m_{1},\left|a_{2}\right|=m_{2}$. Then there exists a constant $d_{c}$ such that if $d>d_{c}$ then $R_{H} \geqslant 0$ everywhere on $S$. Moreover, if $a_{1}$ or $a_{2}$ are different from zero, then $R_{H}>0$ on $S$ for $d>d_{c}$.

Proof. If $a_{1}=a_{2}=0$ then the result is trivial since $R_{\tilde{H}}=0$ in that case. We assume that $\left|a_{1}\right|=m_{1}$ and $a_{1} \neq 0$. We make no restriction on the parameter $a_{2}$, that is $0 \leqslant\left|a_{2}\right| \leqslant m_{2}$. By the symmetry in 1 and 2 of (28) this assumption will cover all cases. As in the non-extreme case, we use a coordinate system centered at $s_{1}$.

We analyze $K_{1}$ directly. We have that $R_{1}$ is positive outside some balls of large radius $r_{+}$ (because the leading term in the asymptotic expansion (A.14) is positive). As in the previous theorem we chose $d$ such that $d>r_{+}$. From equation (29) it follows that $K_{1}$ is positive outside the ball $B$ of radius $r_{+}$centered at $s_{1}$. To analyze the behavior of $K_{1}$ in the interior of $B$ we use 
the explicit expression for $R_{1}$ given by lemma 3.4 and the explicit form of $\tilde{R}_{1}$ given by (13) and (14) to obtain

$$
K_{1}=\frac{e^{-2 q_{1}}}{\psi_{1}^{4}}\left(\frac{\left|\partial \omega_{1}\right|^{2}}{2 X_{1}^{2}}\left(1-\frac{3 u_{2}}{\psi_{1}}\right)+\frac{8\left|\partial \psi_{1}\right|^{2}}{\psi_{1}^{2}} \frac{u_{2}}{\psi_{1}}\right) .
$$

This expression is clearly positive if

$$
\frac{u_{2}}{\psi_{1}}<\frac{1}{3}
$$

By (24) we have that $u_{2}$ is bounded in $B$ and it goes to zero as $d \rightarrow \infty$. Then, condition (31) can always be achieved for sufficiently large $d$. Note that using the upper bound (24) the constant $d_{c}$ can be explicitly calculated. We have proved that $K_{1} \geqslant 0$. To prove that it is strictly positive, we use that $|\partial \omega|^{2}$ only vanishes at the axis (see equations (15)-(17)) and at the axis we can explicitly compute the term $\left|\partial \psi_{1}\right|^{2}$ to see that it is strictly positive.

\section{Final comments}

We have constructed our metric as a sum of two Kerr black hole metrics. It is straightforward to generalize this result to include more than two black holes, that is to superpose more $u_{k}$ and $q_{k}$ in the definition of the conformal metric (5).

We have assumed that the conformal metric is axially symmetric. Due to the particular simpler expression of the scalar curvature of this metric (see equation (28)), this assumption represents a major simplification with respect to the case where the spins point in arbitrary directions. We expect that a result similar to theorem 2.1 remains true in the general case. However, proving such a result requires estimations for many new terms in the scalar curvature and it is not clear how to do this in an efficient way.

Even in the axially symmetric case, it is interesting to see what kinds of properties of the individual metrics are necessary to make the superposition. We have provided sufficient conditions which are satisfied by the Kerr metric. We do not know if these properties are also necessary. Our first attempt was to consider the superposition of two arbitrary Brill metrics (i.e. metrics of the form (2)) with positive Yamabe invariant. But we were unable to prove that an analog of theorem 2.1 holds under these weak assumptions. Inspired by the Kerr data, our next attempt was to impose that the negative part of the Ricci scalar $R^{-}$has compact support. This seems to be a natural assumption, because the support of $R^{-}$seems to play the role of the 'body' and the separation can be taken as the distance between these sets. Furthermore, for Brill metrics the support of $R^{-}$is always nontrivial because the integral of the Ricci scalar over $\mathbb{R}^{3}$ is zero (see [3]). However, for the proof we have also used another particular property of the Kerr metric, namely $u>0$. It is not clear to us whether this condition is actually necessary.

\section{Acknowledgments}

It is a pleasure to thank Robert Geroch for discussions. SD is supported by CONICET (Argentina). GA was partially supported by a 'Conciencias' fellowship, from the Agencia Córdoba Ciencia (Argentina). This work was supported in part by grant PIP 6354/05 of CONICET (Argentina), grant 05/B270 of Secyt-UNC (Argentina) and the Partner Group grant of the Max Planck Institute for Gravitational Physics, Albert-Einstein-Institute (Germany). 


\section{Appendix A. Asymptotic expansions for Kerr initial data}

In this section we study the behavior of Kerr initial data (with parameters $m$ and $a$ ) in the limits $r \rightarrow 0, r \rightarrow \infty$ and $\rho \rightarrow 0$.

The explicit expressions for the functions $q$ and $u$, which characterize the Kerr intrinsic metric (for Boyer-Lindquist slices) $\tilde{h}_{i j}$, in terms of the coordinates $(\rho, z, \phi)$ are given by (see, for example, [13])

$$
\psi^{4}=\frac{X}{\rho^{2}}, \quad e^{2 q}=\frac{\Sigma \sin ^{2} \theta}{X}, \quad u=\psi-1,
$$

where

$$
\Delta=\tilde{r}^{2}+a^{2}-2 m \tilde{r}, \quad \Sigma=\tilde{r}^{2}+a^{2} \cos ^{2} \theta,
$$

and

$$
\begin{aligned}
X & =\left[\frac{\left(\tilde{r}^{2}+a^{2}\right)^{2}-\Delta a^{2} \sin ^{2} \theta}{\Sigma}\right] \sin ^{2} \theta, \\
& =\left(\tilde{r}^{2}+a^{2}+\frac{2 m \tilde{r} a^{2} \sin ^{2} \theta}{\Sigma}\right) \sin ^{2} \theta,
\end{aligned}
$$

where we have defined $\rho=r \sin \theta, z=r \cos \theta$ and

$$
\tilde{r}=r+m+\frac{m^{2}-a^{2}}{4 r} .
$$

The potential $\omega$ which characterizes the Kerr second fundamental form $\tilde{K}_{i j}$ is given by (see, for example, [13])

$$
\omega=2 m a\left(\cos ^{3} \theta-3 \cos \theta\right)-\frac{2 m a^{3} \cos \theta \sin ^{4} \theta}{\Sigma} .
$$

In the following $\tilde{R}$ denotes the Ricci scalar of $\tilde{h}_{i j}$ and $R$ the Ricci scalar of the conformal metric $h_{i j}$ defined by (2).

In the limit $a=0$ we have Schwarzschild data

$$
\psi=1+\frac{m}{2 r}, \quad q=0, \quad R=0, \quad \tilde{R}=0 .
$$

In the following, we assume $a \neq 0$. We analyze first the non-extreme case $|a|<m$. In this case, $u$ has the following asymptotic behavior:

$$
\begin{array}{ll}
u=\frac{m}{2 r}+\mathcal{O}\left(r^{-2}\right) \quad \text { as } \quad r \rightarrow \infty, \\
u=\frac{\sqrt{m^{2}-a^{2}}}{2 r}+\mathcal{O}(1) \quad \text { as } \quad r \rightarrow 0 .
\end{array}
$$

The scalar curvature $R$ satisfies

$$
\begin{aligned}
& R=\frac{2 a^{2}}{r^{4}}+\mathcal{O}\left(r^{-5}\right) \quad \text { as } \quad r \rightarrow \infty, \\
& R=2\left(\frac{4 a}{m^{2}-a^{2}}\right)^{2}+\mathcal{O}\left(r^{2}\right) \quad \text { as } \quad r \rightarrow 0 .
\end{aligned}
$$

10 
For the extreme case $|a|=m$ we have that at infinity $u$ has the same behavior as in the non-extreme case, but at the origin this changes to

$$
u=\frac{\sqrt{2 m}}{\sqrt{r}\left(1+\cos ^{2} \theta\right)^{1 / 4}}+\mathcal{O}(1) \quad \text { as } \quad r \rightarrow 0 .
$$

The behavior of the scalar curvature $R$ and $\tilde{R}$ in this case is given by

$$
\begin{aligned}
& R=\frac{2 m^{2}}{r^{4}+\mathcal{O}\left(r^{-5}\right) \quad \text { as } \quad r \rightarrow \infty,} \\
& R=16\left[\frac{3 \cos ^{2} \theta-1}{\left(1+\cos ^{2} \theta\right)^{4}}\right] \frac{1}{r^{2}}+\mathcal{O}\left(r^{-1}\right) \quad \text { as } \quad r \rightarrow 0
\end{aligned}
$$

and

$$
\tilde{R}=\frac{2 \sin ^{2}(\theta)}{m^{2}\left(1+\cos ^{2}(\theta)\right)}+\mathcal{O}(r) \quad \text { as } \quad r \rightarrow 0 .
$$

Finally, we analyze the behavior of $R$ near the axis $\rho=0$. For a Brill metric like (2), the Ricci scalar is given by [3]

$$
R=-2 e^{-2 q} \bar{\Delta} q,
$$

where $\bar{\Delta}$ is the flat Laplacian in two dimensions, which in coordinates $(r, \theta)$ is given by

$$
\bar{\Delta}=\frac{1}{r} \partial_{r}\left(r \partial_{r}\right)+\frac{1}{r^{2}} \partial_{\theta}^{2} .
$$

The function $q$ defined by (A.1) vanishes at the axis

$$
q(\rho=0, z)=0 .
$$

This can be of course explicitly seen from (A.1). It is also a consequence of the regularity of the metric (2) at the axis (see [3]). As a consequence of (A.18), we have that the radial derivatives of $q$ evaluated at $\rho=0$ (which are equivalent to $z$ derivatives evaluated at $\rho=0$, and hence tangential to the axis where $q$ is constant) vanish. Then, in order to calculate $R$ at the axis using formula (A.16) we need to compute only the derivatives in $\theta$. To calculate them, it is convenient to make the change of variable $\varepsilon=2 \theta$. We have $\partial_{\theta}^{2}=4 \partial_{\epsilon}^{2}$ and $2 \cos ^{2} \theta=1+\cos \varepsilon$. Then we obtain

$$
A:=e^{2 q}=\gamma \frac{(\alpha+\cos \varepsilon)^{2}}{(\beta+\cos \varepsilon)},
$$

where $\gamma, \alpha$ and $\beta$ are functions of $\tilde{r}$ given by

$$
\begin{aligned}
& \alpha:=2 \frac{\tilde{r}^{2}}{a^{2}}+1, \\
& \beta:=2 \frac{\left(\tilde{r}^{2}+a^{2}\right)^{2}}{a^{2} \Delta}-1, \\
& \gamma:=\frac{a^{2}}{2 \Delta} .
\end{aligned}
$$

Calculating the required derivatives we get

$$
\frac{1}{A} \frac{\partial A}{\partial \varepsilon}=-\sin \varepsilon\left[\frac{2}{\alpha+\cos \varepsilon}-\frac{1}{\alpha+\cos \varepsilon}\right]
$$


and

$$
\begin{aligned}
\frac{1}{A} \frac{\partial^{2} A}{\partial \varepsilon^{2}}=-\cos \varepsilon & {\left[\frac{2}{\alpha+\cos \varepsilon}-\frac{1}{\alpha+\cos \varepsilon}\right]+\sin ^{2} \varepsilon\left[\frac{2}{\alpha+\cos \varepsilon}-\frac{1}{\alpha+\cos \varepsilon}\right]^{2} } \\
- & \sin ^{2} \varepsilon\left[\frac{2}{(\alpha+\cos \varepsilon)^{2}}-\frac{1}{(\alpha+\cos \varepsilon)^{2}}\right]^{2}
\end{aligned}
$$

Using (A.24) we finally obtain

$$
\frac{\partial^{2} q}{\partial \varepsilon^{2}}=-\left[\frac{1+\alpha \cos \varepsilon}{(\alpha+\cos \varepsilon)^{2}}\right]+\left[\frac{1+\beta \cos \varepsilon}{(\beta+\cos \varepsilon)^{2}}\right] .
$$

Using (A.16) and (A.25) evaluating at the axis $\varepsilon=0$ we get

$$
R(\rho=0)=\frac{-4}{r^{2}}\left[\frac{1}{1+\beta}-\frac{2}{1+\alpha}\right] .
$$

To prove that this expression is positive, consider the following inequality:

$$
\beta=\frac{2\left(\tilde{r}^{2}+a^{2}\right)^{2}}{a^{2}\left(\tilde{r}^{2}+a^{2}-2 m \tilde{r}\right)}-1>2 \frac{\tilde{r}^{2}}{a^{2}}+1=\alpha,
$$

obtained by replacing the denominator $\left(\tilde{r}^{2}+a^{2}-2 m \tilde{r}\right)$ by $\left(\tilde{r}^{2}+a^{2}\right)$. Note that we assume $m>0$ in order to get the strict inequality. This inequality implies

$$
\frac{1}{1+\beta}-\frac{2}{1+\alpha}<0
$$

and therefore we get our final result

$$
R(\rho=0)>0, \quad \text { on } M .
$$

We emphasize that (A.29) is valid for both the non-extreme and extreme cases.

\section{References}

[1] Baker J G, Centrella J, Choi D-I, Koppitz M and Meter J van 2006 Gravitational wave extraction from an inspiraling configuration of merging black holes Phys. Rev. Lett. 96111102 (arXiv:gr-qc/0511103)

[2] Bartnik R and Isenberg J 2004 The constraint equations The Einstein Equations and Large Scale Behavior of Gravitational Fields ed P T Chruściel and H Friedrich (Basel: Birkhäuser) pp 1-38 (arXiv:gr-qc/0405092)

[3] Brill D 1959 On the positive definite mass of the Bondi-Weber-Wheeler time-symmetric gravitational waves Ann. Phys. 7 466-83

[4] Brill D R and Lindquist R W 1963 Interaction energy in geometrostatics Phys. Rev. 131 471-6

[5] Brugmann B, Gonzalez J A, Hannam M, Husa S and Sperhake U 2008 Exploring black hole superkicks Phys. Rev. D 77124047 (arXiv:0707.0135)

[6] Campanelli M, Lousto C O, Marronetti P and Zlochower Y 2006 Accurate evolutions of orbiting black-hole binaries without excision Phys. Rev. Lett. 96111101 (arXiv:gr-qc/0511048)

[7] Campanelli M, Lousto C O, Zlochower Y and Merritt D 2007 Large merger recoils and spin flips from generic black-hole binaries Astrophys. J. 659 L5-8 (arXiv:gr-qc/0701164)

[8] Cantor M and Brill D 1981 The Laplacian on asymptotically flat manifolds and the specification of scalar curvature Compos. Math. 43 317-30

[9] Choquet-Bruhat Y, Isenberg J and York J W (Jr) 1999 Einstein constraint on asymptotically Euclidean manifolds Phys. Rev. D 61084034 (arXiv:gr-qc/9906095)

[10] Dain S 2001 Initial data for a head on collision of two Kerr-like black holes with close limit Phys. Rev. D 64124002 (arXiv:gr-qc/0103030)

[11] Dain S 2001 Initial data for two Kerr-like black holes Phys. Rev. Lett. 87121102 (arXiv:gr-qc/0012023)

[12] Dain S 2006 Proof of the (local) angular momemtum-mass inequality for axisymmetric black holes Class. Quantum Grav. 23 6845-55 (arXiv:gr-qc/0511087)

[13] Dain S 2006 A variational principle for stationary, axisymmetric solutions of Einstein's equations Class. Quantum Grav. 23 6857-71 (arXiv:gr-qc/0508061) 
[14] Dain S 2008 Proof of the angular momentum-mass inequality for axisymmetric black holes J. Differ. Geom. 79 33-67 (arXiv:gr-qc/0606105)

[15] Dain S, Lousto C O and Zlochower Y 2008 Extra-large remnant recoil velocities and spins from near-extremalBowen-York-spin black-hole binaries Phys. Rev. D 78024039 (arXiv:0803.0351)

[16] González J A, Hannam M, Sperhake U, Brügmann B and Husa S 2007 Supermassive recoil velocities for binary black-hole mergers with antialigned spins Phys. Rev. Lett. 98231101

[17] Hannam M, Husa S, Brügmann B, Gonzalez J A and Sperhake U 2007 Beyond the Bowen-York extrinsic curvature for spinning black holes Class. Quantum Grav. 24 S15-24 (arXiv:gr-qc/0612001)

[18] Herrmann F, Hinder I, Shoemaker D, Laguna P and Matzner R A 2007 Gravitational recoil from spinning binary black hole mergers Astrophys. J. 661 430-6 (arXiv:gr-qc/0701143)

[19] Koppitz M et al 2007 Getting a kick from equal-mass binary black hole mergers Phys. Rev. Lett. 99041102 (arXiv:gr-qc/0701163)

[20] Maxwell D 2006 Rough solutions of the Einstein constraint equations J. Reine Angew. Math. 590 1-29 (arXiv:gr-qc/0405088)

[21] Pretorius F 2005 Evolution of binary black hole spacetimes Phys. Rev. Lett. 95121101 (arXiv:gr-qc/0507014) 